\title{
CONTROLE DA TUBERCULOSE EM MUNICÍPIO FRONTEIRIÇO: ANÁLISE DA CAPACIDADE INSTITUCIONAL DOS SERVIÇOS DE SAÚDE*
}

\author{
Regiane Bezerra Campos ${ }^{1}$, Reinaldo Antonio Silva-Sobrinho², Maria Eugênia Firmino Brunello ${ }^{3}$, \\ Adriana Zilly ${ }^{4}$, Pedro Fredemir Palha ${ }^{5}$, Tereza Cristina Scatena Villa ${ }^{6}$
}

\begin{abstract}
RESUMO: Objetivo: analisar a Atenção Primária à Saúde quanto ao controle da tuberculose em Foz do Iguaçu, Paraná Método: estudo transversal, tipo inquérito, com abordagem quantitativa das dimensões de organização da Atenção à Tuberculose, articulação com a comunidade, autocuidado apoiado, suporte à decisão e desenho do sistema de prestação de serviços, envolvendo 105 profissionais da saúde de 14 unidades no período de dezembro de 2013 a janeiro de 2014 e no ano de 2015 com a análise descritiva e de variância Anova. Resultados: evidenciou-se como pior desempenho a dimensão de articulação com a comunidade, classificada como básica, que obteve média 3,2 e desvio-padrão de 1,8. O melhor desempenho referiu-se à dimensão de autocuidado apoiado, classificada como razoável, com média de 6,9 e desvio-padrão de 2,1. Conclusão: foi observada a necessidade de capacitação dos profissionais e observância aos princípios e diretrizes dos programas e políticas de controle da tuberculose na atenção primária à saúde.
\end{abstract}

DESCRITORES: Atenção primária à saúde; Tuberculose; Áreas de fronteira; Políticas públicas de saúde.

\section{CONTROL OF TUBERCULOSIS IN A BORDER CITY: ANALYSIS OF THE INSTITUTIONAL CAPACITY OF HEALTH SERVICES}

Objective: to analyze primary health care services regarding the control of tuberculosis in the city of Foz do Iguaçu, Paraná. Method: a cross-sectional study, of survey type, with quantitative approach aimed to assess the institutional capacity of the following dimensions of health care services to control TB: organization of the treatment of tuberculosis, coordination with the community, support to self-care, support to decision and design of the service delivery system, involving 105 health professionals from 14 units from December 2013 to January 2014 , and in 2015 with the use of descriptive statistics and Anova analysis. Results: The worst capacity was that of the dimension coordination with the community, classified as low, with a mean of 3.2 and a standard deviation of 1.8. The highest rated dimension was support to self-care, classified as regular, with a mean of 6.9 and a standard deviation of 2.1. Conclusion: need for training of health professionals and compliance with the principles and guidelines of TB control programs and policies in primary health care were identified.

KEYWORDS: Primary health care; Tuberculosis; Border areas; Public health policies.

\section{CONTROL DE TUBERCULOSIS EN MUNICIPIO FRONTERIZO: ANÁLISIS DE CAPACIDAD INSTITUCIONAL DE LOS SERVICIOS DE SALUD}

RESUMEN Objetivo: Analizar la Atención Primaria de Salud en el control de tuberculosis en Foz de Iguaçu, Paraná. Método: Estudio transversal, tipo encuesta, con abordaje cuantitativo de dimensiones de organización de Atención de la Tuberculosis, articulación con la comunidad, autocuidado respaldado, apoyo a la decisión y diseño del sistema de prestación de servicios involucrando a 105 profesionales de salud de 14 unidades, de diciembre de 2013 a enero de 2014, y durante 2015 con análisis descriptivo y de varianza Anova. Resultados: Obtuvo peor desempeño la dimensión de articulación con la comunidad, clasificada como básica, con promedio 3,2 y desvío estándar de 1,8. El mejor desempeño correspondió a la dimensión de autocuidado respaldado, clasificada como razonable, con promedio 6,9 y desvío estándar de 2,1. Conclusión: Se determinó necesidad de capacitación de profesionales y observancia de los principios y directrices de programas y políticas de control de tuberculosis en atención primaria de salud.

DESCRIPTORES: Atención Primaria de Salud; Tuberculosis; Áreas Fronterizas; Políticas Públicas de Salud.

*Artigo extraído da dissertação intitulada: Atenção à tuberculose em um município de tríplice fronteira internacional: o ensino como perspectiva para reorientação da assistência. Ủniversidade do Oeste do Paraná, 2016.

${ }^{1}$ Enfermeira. Doutoranda em Enfermagem em Saúde Pública Escola de Enfermagem de Ribeirão Preto. Universidade de Saúde Paulo. Docente da Universidade Estadual do Oeste do Paraná. Foz do Iguaçu, PR, Brasil.

${ }^{2}$ Enfermeiro. Doutor em Ciências. Docente da Universidade Estadual do Oeste do Paraná. Coordenador de Programa de PósGraduação. Foz do Iguaçu, PR, Brasil.

${ }^{3}$ Enfermeira. Doutora em Ciências. Grupo de Estudos Epidemiológicos e Operacionais em Tuberculose. Escola de Enfermagem de Ribeirão Preto. Universidade de Saúde Paulo. Ribeirão Preto, SP, Brasil.

${ }^{4}$ Bióloga. Doutora em m Ciências Biológicas. Docente da Universidade Estadual do Oeste do Paraná. Foz do Iguaçu, PR, Brasil.

${ }^{5}$ Enfermeiro. Doutor em Enfermagem. Docente da Universidade de Saúde Paulo. Ribeirão Preto, SP, Brasil.

${ }^{6}$ Enfermeira. Doutora em Enfermagem. Docente da Universidade de Saúde Paulo. Ribeirão Preto, SP, Brasil. 


\section{INTRODUÇÃO}

A tuberculose (TB) é considerada uma das principais doenças a serem enfrentadas no mundo. A partir de 2003, essa endemia tornou-se prioridade para o Ministério da Saúde no Brasil, resultando no desenvolvimento de atividades e estratégias para o fortalecimento da Atenção Primária à Saúde (APS) como elemento para a ampliação do acesso e melhoria na qualidade do atendimento às pessoas com $\mathrm{TB}^{(1)}$. Esse país exibe uma distribuição espacial heterogênea de TB nas diferentes regiões brasileiras, com variabilidade de incidência de 11 a 68,4 casos por 100 mil habitantes ${ }^{(2)}$.

O estado do Paraná tem apresentado taxas elevadas de incidência de TB: em 2014, as cifras foram inferiores às registradas em todo o país: no Paraná 18,7 por 100 mil habitantes e no Brasil 33,5 por 100 mil habitantes ${ }^{(2-3)}$. O Sistema de Informação de Agravos de Notificação registrou 2.402 novos casos de TB nesse estado, conforme os dados do Boletim Epidemiológico de 2015, com taxa de incidência de 18,9 casos por 100 mil habitantes e 32,4 por 100 mil habitantes no Brasil, evidenciando assim a diminuição da taxa de incidência no Brasil e o aumento nesse estado ${ }^{(3-4)}$.

Um dos municípios indicados pelo Ministério da Saúde como prioridade para o controle da TB no Paraná localiza-se na Tríplice Fronteira, composta pelas cidades de Foz do Iguaçu-Brasil, Cidade de Leste-Paraguai e Porto Iguaçu-Argentina. Em 2015, Foz do Iguaçu, no estado do Paraná, apresentou o coeficiente de incidência mais elevado (62,9 casos por 100 mil habitantes) quando comparado ao estado (18,9 casos por 100 mil habitantes) e à capital paranaense $\left(17,6\right.$ casos por 100 mil habitantes) ${ }^{(3-4)}$.

Ao se considerar a mobilidade intensa de pessoas, essa região torna-se vulnerável representando um grande desafio para o controle da $\mathrm{TB}^{(5)}$. Reitera-se a existência de iniciativas para o desenvolvimento de ações e serviços sanitários nos municípios de fronteira; entretanto, as políticas de saúde não contemplam as especificidades locais, pois estas se mantêm à margem das políticas centrais de desenvolvimento, representando um território transfronteiriço de intercâmbio e integração ${ }^{(6)}$. Como exemplo, o município de Foz do Iguaçu iniciou o tratamento da doença em pessoas que residem no Paraguai e na Argentina, mas devido à falta de integração dos serviços de saúde, muitas não retornam para o acompanhamento mensal, contribuindo com a elevação do percentual de casos em abandono.

Diante da recente descentralização das ações de controle e tratamento da TB em Foz do Iguaçu, este estudo objetivou analisar a capacidade institucional dos serviços de saúde para o controle da TB no município, tendo como eixo de análise a integração e coordenação das ações como instrumento de gestão dos serviços e ações ofertadas para o tratamento da TB, na perspectiva de tríplice fronteira internacional.

\section{- MÉTODO}

Trata-se de um estudo epidemiológico transversal, tipo inquérito, com abordagem quantitativa. Esta pesquisa foi realizada em Foz do Iguaçu, PR, cidade localizada na tríplice fronteira do Brasil, do Paraguai e da Argentina, que em 2012 possuía população estimada de 255.718 habitantes $^{(7)}$.

A atenção à saúde nesse município está organizada em cinco distritos sanitários (norte, sul, leste, oeste nordeste), compostos por quatro Centros de Referência da Família (CRF), estabelecimentos de saúde da APS que contam com atendimento especializado e equipes multiprofissionais; oito Unidades Básicas de Saúde (UBS), sendo uma delas referência para estrangeiros; e 16 Unidades de Saúde da Família (USF), com 38 equipes. Contam ainda com Unidade de Pronto Atendimento (UPA), Serviços de Emergência e Urgência, Hospital Municipal, Hospitais privados, Centro de Especialidades Médicas e de Referência.

As ações de controle da TB, como o Tratamento Diretamente Observado (TDO), são realizadas na APS pela equipe de referência da área de abrangência, geralmente composta pelo enfermeiro, auxiliar/ técnico de enfermagem e Agentes Comunitários de Saúde (ACS). Os casos mais graves são acompanhados com auxílio da equipe de suporte técnico e complementar em tuberculose (equipe de matriciamento). 
A população de referência foi composta pelos profissionais da equipe mínima para a estratégia saúde da família, registrados no Cadastro Nacional de Estabelecimentos de Saúde (CNES) do município. Como critérios de inclusão elegeram-se profissionais que haviam acompanhado o tratamento de pelo menos um doente de TB em um período mínimo de seis meses anterior à coleta de dados e que estivesse em atividade laboral. Os critérios de exclusão adotados foram os profissionais que apresentavam duplicidade de vínculo, que trabalhavam nos serviços especializados (Ambulatório de Referência, Serviço de Atendimento Especial, Centro de Apoio Psicossocial e Programa de Controle da Tuberculose) e aqueles que trabalhavam em estabelecimentos hospitalares e clínicas particulares.

No cálculo da amostra de profissionais entrevistados levou-se em conta o total de trabalhadores que atuavam nos serviços de APS do município, sendo excluídos os cadastros em duplicidade e os que não atendiam aos critérios de inclusão. Após esse levantamento, procedeu-se ao cálculo do tamanho da amostra com a equação $n_{0}=\frac{P(1-P) Z^{2}}{t^{2}}$, com os seguintes parâmetros: erro amostral de 0,05; intervalo de confiança de $95 \%$ e $\mathrm{P}$ (propôrção populacional) de 50\%. Por ter uma população finita de 252 profissionais, a amostra calculada foi corrigida utilizando a equação ${ }_{n=\frac{n_{0}}{1+\left(n_{0}-1 / N\right)}}$ de tal forma que se obteve o número amostral de 113 (100\%) profissionais.

Dos 113 profissionais do número amostral, a quantidade de profissionais a ser entrevistado de cada categoria foi definida por meio de partilha proporcional, sendo $11(9,75 \%)$ técnicos de enfermagem, 14 $(12,4 \%)$ médicos, 17 (15,05\%) enfermeiros, 19 (16,8\%) auxiliares de enfermagem e 52 (46\%) agentes comunitários de saúde (ACS).

Aceitaram participar da pesquisa 105 (100\%) profissionais de saúde, distribuídos em 10 (62,5\%) USF, três $(37,5 \%)$ UBS e um (25\%) CRF, somando 14 (50\%) estabelecimentos de APS. Destes, 65 (61,9\%) pertenciam à USF, 29 (27,6\%) à UBS e 11 (10,5\%) ao Centro de Referência (CRF).

A coleta de dados e a observação da organização e do processo de trabalho nas unidades de saúde foram realizadas por pesquisadores treinados e ocorreram no período de dezembro de 2013 a janeiro de 2014 e no ano de 2015. Utilizou-se um questionário proposto pelo MacCooll Institute for Health Care Innovation, validado no Brasil por Moyses (2012) e adaptado para a avaliação do controle da tuberculose ou para a avaliação da capacidade institucional dos serviços para o controle da TB por Villa (2012). Esse questionário compreende 7 dimensões: Organização da Atenção à Tuberculose (6 componentes), Articulação com a comunidade (4 componentes), Autocuidado apoiado (4 componentes), Suporte à decisão (4 componentes), Desenho do sistema de prestação de serviços (4 componentes), Sistemas de informação clínica (6 componentes) e Integração dos componentes do modelo de atenção às pessoas com TB (6 componentes). Os componentes avaliativos de cada dimensão possuíam escala de resposta de 0 a 11 , sendo 0 a pior classificação e 11 a melhor.

A dimensão de Organização da Atenção à Tuberculose versa sobre a efetividade do manejo de políticas/programas de controle da tuberculose em todo o sistema (organização, instituição ou unidade) no qual a atenção é prestada, devendo estar orientada e permitir maior ênfase no cuidado aos portadores da doença.

A dimensão de Articulação com a comunidade tratou da articulação entre os sistemas de saúde (instituições ou unidades de saúde) considera também os recursos comunitários, tendo em vista seu importante papel na gestão/manejo clínico da tuberculose.

A dimensão de Autocuidado apoiado busca identificar o autocuidado apoiado e efetivo para pessoas portadoras de TB e suas famílias para lidar com os desafios de conviver e tratar a doença, além de reduzir as complicações e os sintomas.

A dimensão de Suporte à decisão trata do acesso dos profissionais de saúde às informações baseadas em evidência para apoiar decisões na atenção às pessoas usuárias. Isso inclui diretrizes e protocolo fundamentado em evidências, consulta com especialistas, educadores de saúde e o envolvimento dos usuários, de modo a tornar as equipes de saúde capazes de identificar as estratégias efetivas de cuidado.

A dimensão de Desenho do sistema de prestação de serviços focalizou as necessárias mudanças na organização do sistema, realinhando a oferta de cuidado, considerando que a evidência sugere que a gestão efetiva da atenção à TB envolve mais que a simples adição de intervenção a um sistema focado no cuidado de condições agudas. 
A dimensão de Sistemas de informação clínica considera a informação útil e oportuna, individualizada e por populações usuárias de portadores de TB. É um aspecto crítico de modelos de atenção efetivos, especialmente aqueles que empregam abordagens populacionais.

A dimensão de Integração dos componentes do modelo de atenção às pessoas com TB busca evidência sobre o Sistema de Saúde efetivo, se este integra e combina todos os elementos do modelo, por exemplo, associando as metas de autocuidado com os registros nos sistemas de informação, ou associando políticas locais com atividades dos planos de cuidado dos usuários.

Utilizou-se para a análise dos dados o software Statistica 9.0 da Statsoft (Statsoft Inc.). Inicialmente, realizou-se análise descritiva, calculando a média e o respectivo desvio-padrão, de maneira que a capacidade dos serviços de saúde para o controle da TB em cada componente avaliado foi classificada segundo os valores obtidos: entre 0 e 2, capacidade limitada; entre 3 e 5, básica; entre 6 e 8, razoável; e entre 9 e 11, ótima.

Posteriormente, para verificar as diferenças entre as médias, segundo o tipo de serviço de saúde (UBS/USF/CRF), aplicou-se a análise de variância Anova e teste de comparação múltipla, uma vez que atendiam aos pressupostos de homocedasticidade e normalidade pelo teste de Levene e teste de Kolmogorov-Smirnov, respectivamente. O nível de significância estatística adotado para esses testes foi de $5 \%$.

Foi utilizado um roteiro observacional estruturado para registro da organização dos serviços de saúde, bem como o processo de trabalho das equipes para o acompanhamento de casos da TB, os quais deram sustentação para a discussão dos resultados encontrados neste estudo. A pesquisa foi submetida ao

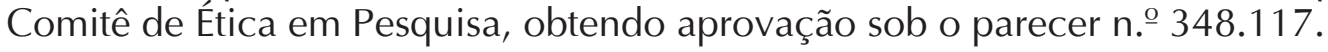

\section{RESUltADOS}

A organização da atenção à TB no município foi classificada com capacidade razoável, 10 (71,4\%) USF exibiram resultado razoável e superior a três $(21,4 \%)$ UBS e um $(7,2 \%)$ CRF, que apresentaram capacidade básica, com 5\% de significância (Tabela 1). Com relação aos componentes de organização da atenção à TB, pode-se observar que benefícios e incentivos aos portadores de TB foram os componentes com menor capacidade na dimensão, classificados como limitados, ou seja, a oferta desses itens é incipiente. Os demais componentes avaliados demonstraram capacidade razoável.

Os resultados revelam que a dimensão articulação com a comunidade em oito (57,1\%) das Unidades de Saúde possui capacidade básica. Ao analisar por tipo de serviço, a USF e UBS tiveram resultados melhores quando comparados ao CRF, que apresentou capacidade limitada; porém, não foi observada diferença estatística significante (Tabela 1).

A dimensão autocuidado apoiado foi a melhor analisada, com capacidade razoável, reproduzindo o mesmo resultado em 13 (92,9\%) das unidades de saúde. As USF apresentaram melhor capacidade nessa dimensão quando comparadas a outros tipos de serviço (Tabela 1).

Quanto ao suporte à decisão, notou-se capacidade básica para o município, UBS e CRF. A USF apresentou capacidade razoável, sendo a melhor em comparação aos demais serviços de saúde (UBS e CRF), com diferença estatística significante (Tabela 1).

O desenho do sistema de prestação de serviços foi classificado como razoável, com a segunda melhor capacidade, observado também em $12(85,7 \%)$ das Unidades de Saúde analisadas no município. A análise, segundo o serviço de saúde, mostrou melhor capacidade das USF para o controle da TB (Tabela 1).

O sistema de informação clínica foi classificado com capacidade razoável no município. Houve diferenças na classificação entre os tipos de unidades, sendo as UBS com capacidade básica e as demais razoáveis. No entanto, essas diferenças não foram observadas estatisticamente (Tabela 1). No tocante aos seus componentes, observou-se capacidade básica para o retorno de informações; os demais componentes apresentaram capacidade razoável. 
A integração dos componentes do modelo de atenção às pessoas com TB apontou capacidade básica para o município; os serviços de saúde também foram classificados com capacidade básica (Tabela 1). Quando os componentes dessa dimensão foram analisados de forma individual, verificou-se capacidade limitada para o componente programas comunitários (ONGs, centros comunitários, igrejas e pastorais), seguido da capacidade básica para os componentes de informação aos portadores de TB e planejamento da atenção.

Tabela 1 - Dimensões de avaliação da capacidade institucional dos serviços para o controle da TB, segundo o município e o tipo de serviço de saúde. Foz do Iguaçu, PR, Brasil, 2014

\begin{tabular}{|c|c|c|c|c|c|c|c|c|c|c|c|c|c|}
\hline DIMENSÕES DE AVALIAÇÃO & & $\begin{array}{ll}\text { NICÍf } \\
\text { ل: } 105\end{array}$ & (1O & & & $\begin{array}{l}\text { USF } \\
\text { v: } 65)\end{array}$ & & & $\begin{array}{l}\text { UBS } \\
\text { v: } 29)\end{array}$ & & & $\begin{array}{ll}\text { CRF } \\
\text { I: 11) }\end{array}$ & \\
\hline Categoria de Análise & $M$ & $D P$ & $C$ & & $M$ & $D P$ & $C$ & $M$ & $D P$ & $C$ & $M$ & $D P$ & $C$ \\
\hline Organização da Atenção à Tuberculose & 6,2 & 1,5 & $\mathrm{R}$ & & $6,5^{a}$ & 1,4 & $\bar{R}$ & $5,6^{b}$ & 1,4 & B & $5,5^{\mathrm{b}}$ & $5,5^{\mathrm{b}}$ & B \\
\hline Articulação com a comunidade & 3,2 & 1,8 & B & & $3,4^{\mathrm{a}}$ & 1,9 & B & $3,2^{\mathrm{a}}$ & 1,8 & B & $2,3^{\mathrm{a}}$ & 1,3 & $\mathrm{~L}$ \\
\hline Autocuidado apoiado & 6,9 & 2,1 & $\mathrm{R}$ & & $7,2^{\mathrm{a}}$ & 1,9 & $\bar{R}$ & $6,1^{\mathrm{a}}$ & 2,3 & $\mathrm{R}$ & $6,7^{\mathrm{a}}$ & 2,4 & $\mathrm{R}$ \\
\hline Suporte à decisão & $\overline{5,8}$ & 2,1 & B & & $6,3^{\mathrm{a}}$ & $\overline{1,7}$ & $\bar{R}$ & $5^{b}$ & 2,4 & B & $4,8^{\mathrm{b}}$ & 2,8 & B \\
\hline $\begin{array}{l}\text { Desenho do sistema de prestação de } \\
\text { serviços }\end{array}$ & 6,6 & 1,7 & $\mathrm{R}$ & & $6,4^{a}$ & 1,6 & $R$ & $6,6^{\mathrm{a}}$ & 1,9 & $R$ & $7,2^{\mathrm{a}}$ & 1,8 & $\mathrm{R}$ \\
\hline Sistemas de informação clínica & 6,3 & 1,8 & $\mathrm{R}$ & & $6,6^{\mathrm{a}}$ & 1,5 & $\mathrm{R}$ & $5,7^{\mathrm{a}}$ & 2 & B & $6,4^{\mathrm{a}}$ & 2,3 & $\mathrm{R}$ \\
\hline $\begin{array}{l}\text { Integração dos componentes do modelo } \\
\text { de atenção às pessoas com TB }\end{array}$ & 5,3 & 1,6 & 105 & B & $5,5^{a}$ & 1,5 & B & $5^{a}$ & 2 & $B$ & $5,5^{\mathrm{a}}$ & 1,8 & $B$ \\
\hline
\end{tabular}

Fonte: Dados da pesquisa, 2014

Legenda: (M) Média; (DP) Desvio-padrão; (C) Classificação.

Interpretação dos resultados: entre 0 e 2 (L), capacidade limitada; entre 3 e 5 (B), capacidade básica; entre 6 e 8 (R), capacidade razoável; e entre 9 e $11(\mathrm{O})$, capacidade ótima. Letras diferentes possuem significância estatística para ANOVA.

\section{- DISCUSSÃO}

A capacidade de organizar a atenção à TB na maioria das UBS e no CRF foi classificada como básica, sendo melhor na USF apontada como razoável. Entretanto, com base no roteiro de observação de campo, foram identificadas diferenças na coordenação do trabalho e fragmentação das ações nas equipes de saúde da família alocadas na mesma unidade de saúde, e observadas fragilidades no processo de trabalho da UBS e CRF, o que poderia ser uma barreira no sentido de melhorar a organização da atenção à TB.

Além disso, outras barreiras da capacidade dos serviços para organizar a atenção à TB na APS são a baixa frequência na oferta de incentivos e benefícios, ausência de atividades educativas aos usuários e necessidade de capacitação profissional. Esses resultados demonstram incoerência com estratégias de adesão positiva ao tratamento diretamente observado ao considerar a relação positiva entre a oferta de benefícios/incentivos e adesão ao Tratamento Diretamente Observado (TOD), assim como o papel da educação em saúde e permanente para o controle da $\mathrm{TB}^{(8)}$.

Evidenciaram-se interesse e empenho por parte dos gerentes das unidades de APS no planejamento da atenção frente às especificidades gerenciais locais, particularmente na provisão de recursos humanos e materiais para o tratamento da TB. Nesse sentido, os aspectos organizacionais do serviço de saúde são decisivos na atenção à TB, sugerindo o uso de práticas gerenciais que invistam em recursos estruturais e de formação na $\mathrm{APS}^{(9)}$.

Ainda na dimensão de organização da atenção à TB, ao considerar o componente de benefícios e incentivos aos portadores de TB, os registros do diário de campo apontam que todas as unidades de saúde (exceto UBS referência para estrangeiros) oferecem o TDO, mas sem estratégia de adesão 
adicional, visto que o município não possui rotina de oferecer benefícios/incentivo para melhorar a adesão ao tratamento. Em contrapartida, os profissionais relataram níveis elevados de cobertura de TDO, e somente a UBS de referência para estrangeiros possui cobertura parcial.

Ressalta-se que a UBS de referência para estrangeiros difere das demais, pois atende imigrantes árabes, chineses e principalmente paraguaios e brasiguaios (filhos de brasileiros residentes no Paraguai e filhos de brasileiros nascidos e residentes no Paraguai). Dessa forma, a dispensa de medicamentos está organizada em torno da possibilidade de locomoção do doente de TB até a unidade de saúde, e leva à dispensação de medicamentos por até 30 dias.

No contexto da dimensão de organização da atenção à TB, ao considerar as metas pactuadas e registradas pela unidade de saúde para o controle da TB na área de abrangência, no tocante à cobertura de tratamento supervisionado e às estratégias para melhoria da atenção à TB, essa dispensação prolongada e adaptada aos portadores de TB estrangeiros inviabiliza o cumprimento dessa meta (TDO) e o acompanhamento desses usuários do serviço de saúde. Esse obstáculo ao TDO segue a lógica das dificuldades para diagnósticos e rastreamento dos doentes e comunicantes nessa região de fronteira internacional(5).

O controle da TB em regiões de fronteira consiste em um desafio que necessita de táticas específicas e pontuais envolvendo diferentes atores, tendo em vista as especificidades socioculturais e locais ${ }^{(10)}$.

A capacidade básica verificada na categoria de análise, suporte e decisão, com exceção da USF, relaciona-se com a falta de conhecimento clínico-epidemiológico em TB por parte dos médicos, particularmente na UBS, onde esse profissional geralmente é plantonista e não se sente responsável pela assistência em TB. Assim, o cuidado é assumido pelo enfermeiro, com suporte da equipe matricial. Evidencia-se que até mesmo em USF, poucos médicos se envolvem e se justificam com relatos de insegurança $^{(11)}$. A realidade contextualizada relaciona-se com a carência de atualizações sobre a TB e a baixa frequência dos médicos nas capacitações oferecidas ${ }^{(8,11)}$.

Ao considerar a rotatividade dos profissionais médicos observada neste estudo, especialmente na UBS, e a dificuldade em desenvolver o processo de trabalho com a equipe por esses profissionais, acredita-se que esta rotatividade gera ruptura no processo de trabalho, fragilizando e interrompendo a formação do trabalho em equipe para o controle da $\mathrm{TB}^{(12)}$.

A articulação com a comunidade e o desenho do sistema detêm fragilidades e revelam deficiência do trabalho em equipe e monitoramento das situações da TB na comunidade. Nesse sentido, considera-se que os resultados desta pesquisa mostram-se incongruentes com os atributos essenciais e os derivados da APS, (integralidade, coordenação e orientação familiar) e as recomendações do Ministério da Saúde para o controle da TB, quando analisados à luz dos atributos essenciais da APS e a Estratégia de Saúde da Família ${ }^{(12-13)}$.

A articulação com a comunidade ocorreu somente por parte do ACS. Contudo, o conhecimento do ACS para o manejo da TB é considerado frágil frente à efetividade das ações de controle da TB realizadas por este ator ${ }^{(14-15)}$. Do ponto de vista qualitativo, faz-se necessário questionar se os ACSs estão capacitados para o papel de articulador entre a comunidade e a equipe de APS, embora seu papel seja de suma importância para o controle da $\mathrm{TB}^{(15-16)}$. Acredita-se que o modelo assistencial atual não favorece o trabalho coletivo e articulado por parte do ACS, uma vez que as ações desse profissional estão vinculadas à dimensão técnica e tangencialmente à política ${ }^{(16)}$.

Outro estudo mostra que os profissionais de saúde não envolvem a comunidade em ações de controle da TB e que de fato as famílias e comunidade não são focalizadas nesse processo ${ }^{(16)}$. Evidências apontam que isso pode estar relacionado com um modelo assistencial limitante do desenvolvimento de um trabalho mais coletivo direcionado às reais necessidades da comunidade ${ }^{(16)}$.

Para a melhoria da atenção à saúde nas diferentes condições crônicas, faz-se necessária a organização contínua do sistema de saúde, integrado nos níveis de assistência, focalizado na promoção e conservação da saúde ${ }^{(16)}$. Ademais, considera-se que, além de um processo coletivo e articulado, é importante que a interação dos profissionais com a família, comunidade e portadores de TB contribua para que estes assumam a função central na manutenção de sua saúde, a partir da educação em saúde, do empoderamento, da gestão e manejo do cuidado, garantindo a continuidade da assistência ${ }^{(16-17)}$. 
Quanto ao desenho do sistema, as evidências sugerem que a gestão efetiva da atenção à TB envolve mais que a simples adição de intervenção a um sistema focado no cuidado de condições agudas: são necessárias mudanças em sua organização, realinhando a oferta de cuidado. Ainda nessa dimensão, foi observado no quesito trabalho em equipe que as ações e responsabilidades estão centradas no enfermeiro, sendo este a principal referência para o tratamento de TB.

Outros estudos corroboram que o enfermeiro é o personagem principal na equipe de trabalho, a primeira referência de cuidado e geralmente o profissional que assume a coordenação, lidera as ações de saúde, viabiliza a aproximação entre os atores e o fortalecimento do vínculo ${ }^{(8,18)}$. Todavia, o cuidado na APS deve ser coerente com a integralidade em saúde, por meio da integração das equipes de saúde e da abordagem multiprofissional, ${ }^{(8)}$ tendo em vista que os profissionais têm atribuições específicas de acordo com a categoria profissional na atenção à TB, e é de suma importância que cada profissional conheça suas atribuições na atenção à $\mathrm{TB}^{(19)}$.

Acerca do enfermeiro, estudos que visam à gestão do cuidado em TB mostram que a formação desse profissional precisa melhorar em direção à promoção do cuidado, e não na concepção biomédica, em prática mecanicista e tarefeira ${ }^{(8,20)}$. Ao considerar o conhecimento dos enfermeiros da APS, um estudo realizado em Foz do Iguaçu evidencia a necessidade de aprimoramento para a realização do manejo da TB, já que a própria formação dos profissionais conduz ao fazer técnico e à concepção biologicista. Destaca-se que esse modelo tem predominado sobre o acolhimento, a integralidade no cuidado e os aspectos culturais dos usuários, ${ }^{(21)}$ e que as capacitações disponibilizadas pelos gestores de saúde são insuficientes do ponto de vista quantitativo dos profissionais ${ }^{(8,22)}$.

Assim, sinaliza-se a necessidade de educação permanente dos profissionais da APS, pois o cuidado deve ser baseado na integralidade em saúde por meio da integração das equipes e da abordagem multiprofissional, ${ }^{(18)}$ como também da adequação das ações à realidade local e coerência com as diretrizes para o controle da TB e o uso de metodologias condizentes com a condição de saúde ${ }^{(14,19-22)}$.

O autocuidado apoiado, ou seja, os componentes relacionados com os registros sobre o apoio e suporte dos profissionais na unidade de saúde para o portador de TB cuidar da própria saúde, acolhimento das preocupações dos portadores de TB e familiares e as intervenções de mudança de comportamento de portadores de TB apontaram capacidade razoável; porém, na observação de campo foram identificadas limitações nas iniciativas para o autocuidado apoiado, como ações de educação em saúde e estabelecimento de vínculo com os profissionais da equipe de referência, e intervenções para mudança de comportamento no sentido do portador de TB parar de fumar ou de persistir em outros comportamentos que retardam ou dificultam a recuperação e cura da TB.

Nessa direção, esses resultados corroboram com a observação que a educação em saúde não é uma atividade regular na prática assistencial dos profissionais de saúde. Existem dificuldades na inserção de processos de trabalhos integrados devido à falta de formação/educação permanente, fragilizando o controle da $\mathrm{TB}^{(8)}$.

Entende-se que o modelo de atenção às condições crônicas encoraja o diálogo entre as políticas de saúde e ensino, considerando-se que na gestão dessas condições de saúde admite-se que a mudança de comportamento dos profissionais de saúde e das pessoas usuárias dos serviços de saúde ocorre por meio de processos educacionais robustos voltados a esses atores ${ }^{(16)}$. Portanto, os profissionais de saúde devem ser parceiros nesse processo de empoderamento, e a singularidade dos papéis dos atores (profissionais e usuários) deve ser complementar ${ }^{(16,12)}$.

Embora o sistema de informação clínica tenha revelado capacidade razoável na maioria das unidades de saúde, verificou-se capacidade básica nas UBS e no quesito de retorno das informações para todos os tipos de serviços. Nesse contexto, o prontuário clínico é utilizado pelos três tipos de serviços e inclui registros de diagnóstico, terapêutica, agendamento de retorno e registros de realização de TDO. Entretanto, o Livro de Registro e Acompanhamento de Casos de TB (Livro Verde) não é utilizado por todos os serviços, porque o prontuário eletrônico está em fase de implantação e algumas informações estão sendo alimentadas nesse novo sistema. Similarmente, outros pesquisadores demonstram que profissionais acreditam ser desnecessária a utilização do livro verde, mas pontua-se que esse instrumento pode ser muito útil na avaliação e planejamento das ações no nível local ${ }^{(11)}$. 
A efetiva atenção às condições crônicas depende do acesso às informações individuais e coletivas da população. Para tanto, torna-se necessário um sistema de informação integrado vinculado aos diferentes componentes da rede de atenção, que seja um facilitador da atenção à saúde, que viabilize a elaboração de planos de cuidados e a retroalimentação das informações ${ }^{(16)}$.

Quanto à capacidade de integração dos componentes do modelo de atenção nos serviços de APS, verificou-se a capacidade básica no planejamento da atenção à TB e de oferecer informações sobre o plano de cuidado aos doentes, evidenciando que a integração/comunicação entre as iniciativas comunitárias e as unidades de saúde são limitadas. Além disso, o quesito de articulação com a comunidade demonstra que na prática a capacidade de orientação comunitária e familiar, no contexto da atenção à saúde, enfoca de forma limitada a razoável as famílias e a comunidade em seu processo de trabalho.

Observou-se de maneira muito frágil a integração das ações da equipe de saúde com a comunidade e familiares dos portadores de TB, principalmente nos programas comunitários, seguida do planejamento da atenção à TB na UBS de referência do portador de TB e nas informações aos portadores de TB em relação ao seu plano de cuidado.

Nesse âmbito, acredita-se que o formato de organização dos serviços de atenção à saúde dificulta o vínculo e o atendimento integral aos usuários, decorrente de posturas engessadas oriundas dos profissionais e da desarticulação entre a organização do serviço e a necessidade dos doentes ${ }^{(14,23)}$.

As dificuldades de integração entre os níveis de assistência e os problemas de comunicação e integração estão vinculadas às políticas de saúde seletivas e à atuação do mercado privado em serviços de média e alta tecnologia, os quais prestam serviços pontuais ${ }^{(24)}$.

A limitação deste estudo pode estar relacionada com um possível viés de informação sobre o funcionamento da atenção à TB na APS, uma vez que a descentralização da atenção à TB é recente e existe a possibilidade de alguns profissionais terem respondido às questões a partir de suas experiências pessoais e recomendações técnicas.

\section{CONCLUSÃO}

A atenção à TB no município é descentralizada nas unidades de USF e UBS, sendo contemplada por processos de trabalho assimétricos, fragmentados e desarticulados aos princípios da APS e diferentes entre si.

As dimensões de avaliação utilizadas se mostraram interconectadas e desvelaram fragilidades nos processos de trabalho e problemas estruturais relacionados com a informação, comunicação, integração e suporte à decisão e especialmente com a articulação com a comunidade, levando dificuldade na organização do sistema e coordenação do cuidado.

Recomenda-se para a APS em região de fronteira a atenção à saúde baseada não apenas em seus atributos essenciais e derivados, mas também nas especificidades locais e regionais no contexto de fronteira e que seja orientada para a lógica da atenção às condições crônicas de saúde. Considera-se essencial o envolvimento dos gestores, a proposição de metas/estratégias claras e alcançáveis, como também investimento em recursos humanos, estruturais e materiais para que a APS de fato assuma seu papel efetivo na rede de atenção à saúde, orientada para a lógica da atenção às condições crônicas. Reforça-se a necessidade de comunicação institucionalizada e informatizada, como também o fortalecimento do trabalho em equipe e educação em saúde. 


\section{REFERÊNCIAS}

1. Ministério da Saúde (BR). Secretaria de Vigilância em Saúde. Boletim Epidemiológico: O controle da tuberculose no Brasil: avanços, inovações e desafios. [Internet] Brasília: Ministério da Saúde; 2014 [acesso em 20 mar 2017]. Disponível: http://bvsms.saude.gov.br/bvs/periodicos/boletim_epidemiologico_numero_2_2014.pdf.

2. Ministério da Saúde (BR). Secretaria de Vigilância em Saúde. Boletim Epidemiológico: Detectar, tratar e curar: desafios e estratégias brasileiras frente à tuberculose. [Internet] Brasília: Ministério da Saúde; 2015 [acesso em 20 mar 2017 ] Disponível: http://portalarquivos.saude.gov.br/images/pdf/2015/marco/25/Boletim-tuberculose-2015.pdf.

3. Ministério da Saúde (BR). Ministério da Saúde. Departamento de Informática do SUS. Informação em saúde: Tuberculose - casos confirmados notificados no sistema de informação de agravos de notificação - Paraná. Casos confirmados por ano diagnóstico. Período 2015. [Internet] Brasília: Ministério da Saúde; 2015 [acesso em 10 mar 2017 ]. Disponível: http://tabnet.datasus.gov.br/cgi/tabcgi.exe?sinannet/cnv/tubercPR.def.

4. Ministério da Saúde (BR). Secretaria de Vigilância em Saúde. Boletim Epidemiológico: Indicadores prioritários para o monitoramento do Plano Nacional pelo Fim da Tuberculose como Problema de Saúde Pública no Brasil. [Internet] Brasília: Ministério da Saúde; 2017 [acesso em 09 dez 2017]. Disponível: http://portalarquivos2.saude.gov.br/images/ pdf/2017/marco/23/2017-V-48-N-8-Indicadores-priorit--rios-para-o-monitoramento-do-Plano-Nacional-pelo-Fim-daTuberculose-como-Problema-de-Sa--de-P--blica-no-Brasil.pdf.

5. Silva-Sobrinho RA, Andrade RLP, Ponce MAZ, Wysocki AD, Brunello ME, Scatena LM, et al. Retardo no diagnóstico da tuberculose em município da tríplice fronteira Brasil, Paraguai e Argentina. Rev Panam Salud Publica. [Internet] 2012;31(6) [acesso em 20 fev 2017]. Disponível: http://www.scielosp.org/scielo.php?script=sci_ arttext\&pid=S1020-49892012000600003.

6. Preus LT, Nogueira MR. O pacto pela saúde nas cidades-gêmeas da fronteira do Rio Grande do Sul com a Argentina e o Uruguai. Textos \& Contextos. [Internet] 2012;11(2) [acesso em 08 mar 2017]. Disponível: http://revistaseletronicas. pucrs.br/ojs/index.php/fass/article/view/12346.

7. Instituto Brasileiro de Geografia e Estatística (IBGE). Estimativas de população enviadas ao TCU, 2012. Estimativas da população residente no Brasil e Unidades da Federação, 2012. [Internet] Foz do Iguaçu: IBGE; 2012 [acesso em 09 dez 2017]. Disponível: ftp://ftp.ibge.gov.br/Estimativas_de_Populacao/Estimativas_2012/estimativa_2012_municipios.pdf.

8. de Sá LD, de Oliveira AAV, Gomes ALC, Nogueria JA, Villa TCS, Collet N. Cuidado ao doente de tuberculose na Estratégia Saúde da Família: percepções de enfermeiras. Rev. esc. enferm. USP. [Internet] 2012;46 (2) [acesso em 14 fev 2017]. Disponível: http://dx.doi.org/10.1590/S0080-62342012000200013.

9. Silva-sobrinho RA, Ponce MAS, Andrade RLP, Beraldo AA, Pinto ESG, Scatena LM, et al. Efetividade no diagnóstico da tuberculose em Foz Do Iguaçu, Tríplice fronteira Brasil, Paraguai e Argentina. Rev Esc Enferm USP. [Internet] 2013;47(6) [acesso em 09 dez 2017]. Disponível: http://dx.doi.org/10.1590/S0080-623420130000600018.

10. Belo EN, Orellana JDY, Levino A, Basta PC. Tuberculose nos municipios da frontera Brasil-Colombia-Peru-Venezuela: situação epidemiológica e fatores associados ao abandono. Rev Panam Salud Publica. [Internet] 2013;34(5) [acesso em 10 dez 2017]. Disponivel: https://scielosp.org/scielo.php?script=sci_arttext\&pid=S1020-49892013001100004.

11. Cunha NV, Cavalcanti ML, Costa AJL. Diagnóstico situacional da descentralização do controle da tuberculose para a Estratégia de Saúde da Família em Jardim Catarina- São Gonçalo. Cad. Saude Colet. [Internet] 2012;20(2) [acesso em. 08 fev 2017]. Disponível: http://www.cadernos.iesc.ufrj.br/cadernos/images/csc/2012_2/artigos/csc_v20n2_177-187. pdf.

12. Lopes LMG, Vieira NF, Lana FCF. Análise dos atributos da Atenção Primária à Saúde na atenção à tuberculose no Brasil: uma revisão integrativa. Rev. enferm. Cent.-Oeste Min. [Internet] 2015;5(2) [acesso em 11 dez 2017] Disponível: http://www.seer.ufsj.edu.br/index.php/recom/article/view/678/870.

13. Oliveira MAC, Pereira IC. Atributos essenciais da Atenção Primária e a Estratégia Saúde da Família. Rev Bras Enferm. [Internet] 2013;66(n.esp) [acesso em 05 mai 2017]. Disponível: http://dx.doi.org/10.1590/S0034-71672013000700020. 
14. Crispim JA, da Silva LMC, Pinto IC, Palha PF, Arcênio RA. Agente Comunitário de Saúde no controle da tuberculose na Atenção Primária à Saúde. Acta paul. enferm. [Internet] 2012;25(5) [acesso em 20 fev 2017]. Disponível: http:// dx.doi.org/10.1590/S0103-21002012005000021.

15. Sobrinho ECR, de Freitas KG, de Figueiredo RM, Caliari JS. A tuberculose na estratégia de saúde da família: o conhecimento dos agentes comunitários de saúde. Rev Eletr Enf. [Internet] 2013;15(2) [acesso em 30 jun 2016]. Disponível: http://dx.doi.org/10.5216/ree.v15i2.18046.

16. Scatolin BE, Pinto ESG, Arcêncio RA, Andrade RLP, Wysocki AD, Ponce MAZ, et al. Busca de sintomáticos respiratórios: atuação do Agente Comunitário de Saúde no controle da tuberculose em município de grande porte, Brasil. Texto contexto - enferm. [Internet] 2014;23(2) [acesso em 25 mar 2017]. Disponível: http://dx.doi.org/10.1590/010407072014001600012.

17. de Assis EG, Beraldo AA, Monroe AA, Scatena LM, Cardozo-Gonzales RI, Palha PF et al. Coordenação da assistência no controle da tuberculose. Rev. esc. enferm. USP. [Internet] 2012;46(1) [acesso em 30 mai 2017]. Disponível: http:// dx.doi.org/10.1590/S0080-62342012000100015.

18. Araújo LGP, Saldanha RA, Colonese CR. O enfermeiro e a educação em saúde no atendimento aos portadores de tuberculose (TB) na unidade básica de saúde. Rev. pesqui. cuid. fundam. [Internet] 2014;6(1) [acesso em 11 dez 2017]. Disponível: http://dx.doi.org/10.9789/2175-5361.2014v6n1p378.

19. dos Santos TMMG, Nogueira LT, Arcencio RA. Atuação de profissionais da Estratégia Saúde da Família no controle da tuberculose. Acta paul. enferm. [Internet] 2012;25(6) [acesso em 20 jun 2017]. Disponivel: http://dx.doi.org/10.1590/ S0103-21002012000600020.

20. Barrêto AJR, Evangelista ALF, de Sá SAA, de Almeida SA, Nogueira JA, Lopes AMC. Gestão do cuidado à tuberculose: da formação à prática do enfermeiro. Rev Bras Enferm. [Internet] 2013;66(6) [acesso em 20 mai 2017]. Disponível: http://dx.doi.org/10.1590/S0034-71672013000600006.

21. Sobrinho RAS, Souza AL, Wysocki AD, Silva LMC, Beraldo AA, Villa TCS. Conhecimento de enfermeiros de unidades de atenção básica acerca da tuberculose. Ver. Cogitare Enferm. [Internet] 2014;19(1) [acesso em 01 jun 207]. Disponível: http://dx.doi.org/10.5380/ce.v19i1.35930.

22. Barbosa GC, Meneguim SL, Molina SA, Moreno V. Política Nacional de Humanização e formação dos profissionais de saúde: revisão integrativa. Rev. bras. enferm. [Internet] 2013;66(1) [acesso em 01 jun 207]. Disponível: http://dx.doi. org/10.1590/S0034-71672013000100019.

23. Ballestero JGA, Moncaio ACS, da Silva LMCS, Surniche CA, de Lima MCRA, Palha PF. Tuberculose multirresistente: integralidade da atenção à saúde na perspectiva discursiva. Esc. Anna Nery. [Internet] 2014;18(3) [acesso em 20 mai 2017]. Disponível: http://dx.doi.org/10.5935/1414-8145.20140073.

24. de Macedo LM, Martin STF. Interdependência entre os níveis de atenção do Sistema Único de Saúde (SUS): significado de integralidade apresentado por trabalhadores da Atenção Primária. Interface (Botucatu). [Internet] 2014;18(51) [acesso em 01 jun 2017]. Disponível: http://dx.doi.org/10.1590/1807-57622013.0597. 